\title{
Third-Order Nonlinear Optical Properties and Reverse Saturable Absorption in 2,3-Butanedione Dihydrazone Using $z$-Scan Technique
}

\author{
V. Singh ${ }^{a, b}$, P. AghamKAR ${ }^{c, *}$ AND B. LAL ${ }^{d}$ \\ ${ }^{a}$ Department of Applied Physics, Guru Jambheshwar University of Science and Technology \\ Hisar-125001, Haryana, India \\ ${ }^{b}$ Department of Applied Science, N.C. College of Engineering, Israna, Panipat-132107, Haryana, India \\ ${ }^{c}$ Department of Physics, Chaudhary Devi Lal University, Sirsa-125055, Haryana, India \\ ${ }^{d}$ Department of Applied Science, Government Polytechnic for Women, Sirsa-125055, Haryana, India
}

(Received January 21, 2012; in final form December 20, 2012)

Using chemical route a 2,3-butanedione dihydrazone (BDDH) compound was prepared. The third-order nonlinear optical properties of BDDH were investigated by using single beam $z$-scan technique with $Q$-switched, frequency doubled Nd:YAG laser $(\lambda=532 \mathrm{~nm})$ at $5 \mathrm{~ns}$ pulse. Effect of excitation intensity on nonlinear optical properties has been studied and found that experimental and computed values are well in agreement. Also, we found the measured nonlinear optical coefficients are nearly ten times larger than the reported. The excited state absorption cross-section of BDDH was found to be larger than that of the ground state absorption cross-section, indicating the reverse saturable absorption.

DOI: 10.12693 /APhysPolA.123.39

PACS: 42.65.-k, 42.70.Mp, 42.70.Nq

\section{Introduction}

Nonlinear optical materials exhibiting a large third-order optical nonlinearity are in great demand because of their functional applications in optical power limiting [1-3], optical switching [4-6], optical data storage $[7,8]$, microfabrication [9] and optical phase conjugation [10-12]. Among various third-order nonlinear optical materials, organic materials are promising candidate materials due to their synthetic flexibility, high damage resistance and a large optical nonlinearity [13]. In organic materials, conjugate systems have received much attention as they show high density polarizable $\pi$-electrons along the backbone and electron donating/accepting character [14-17]. 2,3-butanedione dihydrazone (BDDH) is one of the important molecules of $\pi$-electron conjugate system and it consists of an alternate pair of nitrogen and carbon atoms and electron donating amino group at both end of the chain. The presence of pair of nitrogen atoms in BDDH provides increased stability towards oxidation and hydrolysis, due to which it is an environmentally stable material $[18,19]$. Beside, nitrogen atoms also serve to modulate the electron density along the chain and leading to charge transfer within the BDDH [20]. These typical features of BDDH, stimulated our interest to synthesize and investigate its third-order nonlinear optical properties for possible functional applications.

Cubic optical nonlinearity has been characterised by various techniques $[13,21]$. These techniques have their

*corresponding author; e-mail: prasut2003@yahoo.co.in own merits and demerits and therefore they occupy a special place in nonlinear optics. Among them, z-scan technique [22] has a great advantage because of its simplicity and high sensitivity by which one can characterize simultaneously nonlinear absorption and refraction of optical materials via open and closed aperture measurements, respectively. In addition, signature of optical nonlinearity can also be identified by this technique. Very recently, third-order nonlinear optical properties of conjugated polymer [23] and thiophene based conjugated polymer [24] have been investigated by two-photon-induced fluorescence method and $z$-scan technique, respectively. Wang et al. [23] showed that the two-photon absorption cross-section of conjugate polymers varies inversely proportional to the concentration of the solvents but independently of polarity of the solvents, while Poornesh et al. [24] pointed out that the origin of a large third-order optical nonlinearity lies in strong delocalization of $\pi$-electrons along the polymer chain. Li et al. [25] investigated third-order nonlinear optical properties with different substituted azobenzene side chain using $z$-scan technique and found that push-pull electronic effect plays important role in enhancing the magnitude of $\chi^{(3)}$ in soluble polymers. Here, we report the third-order nonlinear optical properties of as-prepared BDDH sample by using $z$-scan technique for different excitation intensities with $Q$-switched frequency doubled Nd:YAG laser (532 nm) at $5 \mathrm{~ns}$ pulse. Effect of different excitation intensities on third-order nonlinear optical properties has been studied and found that experiment and theory are well in agreement. 


\section{Theory}

$z$-scan technique is based on the measurement of the nonlinear transmission of the optical materials in open and close aperture configuration. The transmittance is measured in the linear diffraction regime.

\subsection{Open aperture}

In open aperture measurements, the normalized transmittance is given by [22]:

$$
T(z, S=1)=\sum_{m=0}^{\infty} \frac{\left[-q_{0}(z)\right]^{m}}{(m+1)^{3 / 2}} \quad \text { for } q_{0}(z)<1,
$$

where $q_{0}(z)=\frac{\beta I_{0}[1-\exp (-\alpha L)]}{\left(1+z^{2} / z_{0}^{2}\right) \alpha}, \beta$ is nonlinear absorption coefficient, $\alpha$ is the linear absorption coefficient, $I_{0}$ is the on-axis excitation intensity at the focus, $L$ is the length of sample, $z$ is the sample position, $z_{0}=\pi \omega_{0}^{2} / \lambda$ is the Rayleigh range with $\omega_{0}$ being the beam waist radius at the focal point $(z=0)$, and $\lambda$ is the laser wavelength. By knowledge of nonlinear absorption coefficient $(\beta)$, one may also compute corresponding imaginary part of third-order susceptibility $\left(\operatorname{Im} \chi^{(3)}\right)$ by the following relations:

$$
\begin{aligned}
& \operatorname{Im} \chi^{(3)}(\mathrm{SI})=\frac{2 \varepsilon_{0} c^{2} n_{0}^{2} \beta(\mathrm{SI})}{3 \omega}, \\
& \operatorname{Im} \chi^{(3)}(\mathrm{esu})=\frac{9 \times 10^{8} \operatorname{Im} \chi^{(3)}(\mathrm{SI})}{4 \pi},
\end{aligned}
$$

where $\varepsilon_{0}$ is the permittivity of free space, $n_{0}$ is linear refractive index and $c$ is speed of light in vacuum. In addition to $\beta$, the excited-state absorption cross-section $\left(\sigma_{\text {exc }}\right)$ of the sample can also be obtained from the normalized open aperture data by the following standard approach [15]. The change in the intensity of the laser beam, as it propagates through the sample is given by

$$
\frac{\mathrm{d} I}{\mathrm{~d} z}=-\alpha I-\frac{\sigma_{\mathrm{exc}} \alpha I}{\hbar \Omega} \int_{-\infty}^{t} I\left(t^{\prime}\right) \mathrm{d} t^{\prime} .
$$

Here, $N(t)$ is the density of charges in the excited state, $\Omega$ is the angular frequency of laser. By solving Eq. (3) for the different incident energy of the laser over the spatial extent of the beam, the normalized transmittance for open aperture can be given as

$$
T=\ln \frac{\left(1+\frac{q_{0}}{1+x^{2}}\right)}{\left(\frac{q_{0}}{1+x^{2}}\right)},
$$

where

$$
q_{0}=\frac{\sigma_{\mathrm{exc}} \alpha F_{0} L_{\mathrm{eff}}}{2 \hbar \Omega}
$$

is the free parameter, $F_{0}\left(=\frac{2 \xi_{0}}{\pi \omega_{0}^{2}}\right)$ is the on-axis fluence at the focus. $\xi_{0}$ is incident energy. Expression (4b) is used for obtaining the values of $\sigma_{\text {exc }}$ by fitting the open aperture data. However, the ground state absorption cross-section $\left(\sigma_{\mathrm{g}}\right)$ of the sample can be straightforwardly obtained from the relation

$$
\alpha=\sigma_{\mathrm{g}} N_{\mathrm{a}} C,
$$

where $N_{\mathrm{a}}$ is Avogadro's number and $C$ is the concentration in $\mathrm{mol} / \mathrm{cm}^{3}$.

\subsection{Close aperture}

The nonlinear refractive index $\left(n_{2}\right)$ can be obtained by the normalized transmittance of close aperture described by the following relation:

$$
T=1+\frac{4 \Delta \phi_{0} x}{\left(x^{2}+1\right)\left(x^{2}+9\right)},
$$

where $x=z / z_{0}$ and $\Delta \phi_{0}$ is the phase change given by $\Delta \phi_{0}=k n_{2} I_{0} L_{\text {eff }}$, where $k\left(=\frac{2 \pi}{\lambda}\right)$ is wave number and $L_{\text {eff }}$ is the effective length in the sample. The dependence of normalized transmittance on aperture and phase shift is given by [22]:

$$
\Delta T_{P-V}=0.406(1-S)^{0.25}\left|\Delta \phi_{0}\right|,
$$

where $\Delta T_{P-V}$ (denoting $\left.T_{P}-T_{V}\right)$ is the difference between normalised peak and valley transmittance and $S\left(=1-\mathrm{e}^{\left(-2 r_{\mathrm{a}}^{2} / r_{\mathrm{b}}^{2}\right)}\right)$ represent the aperture linear transmittance, where $r_{\mathrm{a}}$ denotes the aperture radius and $r_{\mathrm{b}}$ denotes the beam radius at the aperture.

Also, by knowledge of index of nonlinear refraction one may obtain corresponding real part of third-order optical susceptibility using the following relations:

$$
\begin{aligned}
& \operatorname{Re} \chi^{(3)}(\mathrm{SI})=\frac{4 \varepsilon_{0} c n_{0}^{2} n_{2}(\mathrm{SI})}{3}, \\
& \operatorname{Re} \chi^{(3)}(\mathrm{esu})=\frac{9 \times 10^{8} \operatorname{Re} \chi^{(3)}(\mathrm{SI})}{4 \pi} .
\end{aligned}
$$

Finally using Eqs. (2) and (8), magnitude of $\chi^{(3)}$ can be obtained as: $\chi^{(3)}=\left|\left(\operatorname{Re}\left(\chi^{(3)}\right)\right)^{2}+\left(\operatorname{Im}\left(\chi^{(3)}\right)\right)^{2}\right|^{1 / 2}$.

\section{Experimental}

\subsection{Sample preparation}

The scheme of synthesis and molecular formula of BDDH is expressed in Fig. 1. 2,3-butanedione dihydrazone sample was synthesised through chemical route [26]. A solution of $30 \mathrm{ml}(0.617 \mathrm{~mol})$ of hydrazine hydrate (Sigma-Aldrich, reagent grade) was dissolved in $300 \mathrm{ml}$ of ethanol with 5 drops of glacial acetic acid (Sigma-Aldrich, $99.9 \%)$ as a catalyst. Then a solution of $12 \mathrm{ml}(0.137)$

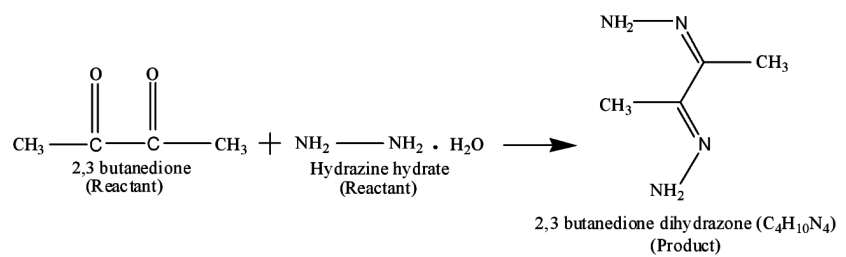

Fig. 1. Synthesis scheme of 2,3-butanedione dihydrazone.

of 2,3-butanedione (Aldrich, 97\%) dissolved in $25 \mathrm{ml}$ of ethanol was added to the above prepared solution dropwise with stirring for one hour. The resultant solution was stirred at room temperature for another two hours, capped and refrigerated overnight and finally white product was obtained after filtering. The final product was washed with cold ethanol and dried under vacuum for 
$10 \mathrm{~h}$. The linear absorption spectrum of BDDH was recorded by using a UV-VIS spectrophotometer (Cary$-5000)$.

\section{2. z-scan experiments}

A schematic sketch of the single beam $z$-scan experimental setup $[22,27]$ is shown in Fig. 2. The sample was irradiated by a $Q$-switched frequency doubled $(\lambda=532 \mathrm{~nm}) \mathrm{Nd}-\mathrm{YAG}$ laser (Quanta system, HYL101) having a 5 ns pulse duration with pulse repetition rate of $10 \mathrm{~Hz}$. The fundamental Gaussian laser beam (verified by knife-edge experiment) was tightly focused to $w_{0}=15 \mu \mathrm{m}$ spot, where $w_{0}$ is the spot size of the beam. Firstly, z-scan experimental setup was calibrated with $\mathrm{CS}_{2}$ and subsequently measurements of third-order nonlinear absorption and refraction coefficients of BDDH were performed.

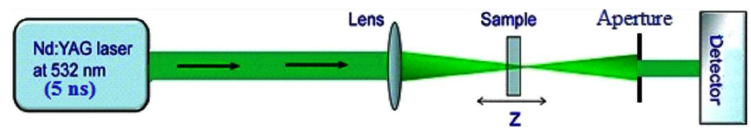

Fig. 2. z-scan experimental arrangement.

In order to investigate nonlinear optical properties of $\mathrm{BDDH}$, as-prepared sample was dissolved in dimethyl sulphoxide $(\approx 10 \mathrm{mM}$ concentration $)$ and kept in a quartz cell (sample holder) of $1.0 \mathrm{~mm}$ path length $(l)$. Thin sample approximation i.e. $l<z_{0}\left(z_{0}=\pi w_{0}^{2} / \lambda\right.$ is the Rayleigh range) was ensured. The sample holder was mounted on a $x y z$ translation stage (THORLABS-PT$-3-1 ")$ and the transmitted pulse energy was collected by a detector (Thorlab DET 110). The output energy recorded by $200 \mathrm{MHz}$ digital storage oscilloscope (Tektronics TDS 2024). Open and closed aperture configurations were used for measuring nonlinear absorption and refraction of the sample, respectively. For closed aperture measurement, an aperture with linear aperture transmittance value $S=0.015$ was placed in front of transmission detector and the transmittance was recorded as a function of sample position along the $z$-axis. In order to study the effect of excitation intensity on nonlinear optical response of BDDH open and close aperture measurements were performed at different excitation intensities $0.4,1.01,2.14$, and $3.31 \mathrm{GW} / \mathrm{cm}^{2}$. We noticed that scattering and thermal effects were very weak around these excitation levels and hence these effects were neglected without losing the generality of the problem [28]. Moreover, low repetition rate of the laser allows one to neglect the contribution of pulse-to-pulse build up effect in nonlinear optical response of BDDH [29].

\section{Results and discussion}

The optical absorption spectrum of BDDH shows a well developed broad peak at $290 \mathrm{~nm}$ and transmission cut-off around $350 \mathrm{~nm}$. In Fig. 3, linear absorption ranging from $250 \mathrm{~nm}$ to $350 \mathrm{~nm}$, indicates the transition between $n-\pi^{*}$ and $\pi-\pi^{*}$ orbital and is responsible for occurrence of a large value of third-order nonlinearity in organic materials $[20,30]$.

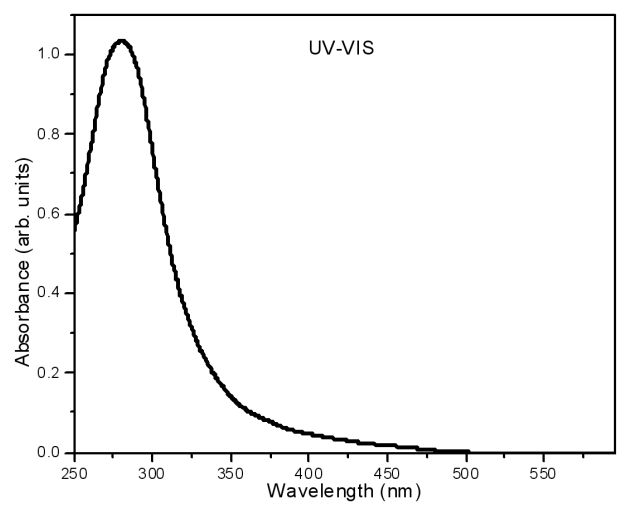

Fig. 3. Optical absorption spectrum of 2,3-butanedione dihydrazone.

An open aperture transmission of the sample was measured in the far-field region. A decrease in value of transmittance around the focal point, is indicative of nonlinear absorption in BDDH (see Fig. 4). In this figure, symbols represent experimental transmission data, while solid lines are obtained by fitting the experimental data to the nonlinear transmittance given by Eq. (1). For theoretical fitting, we assumed $q_{0}(0)<1$ and $\beta$ as a fitting parameter.

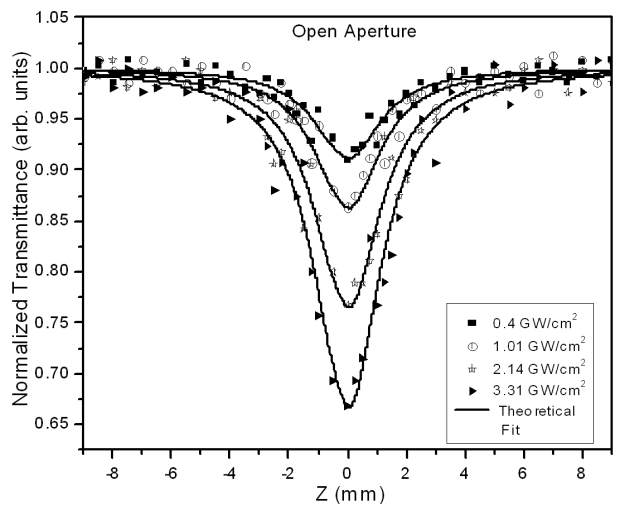

Fig. 4. Open aperture measurements (at $532 \mathrm{~nm}$ ) of 2,3-butanedione dihydrazone at different excitation intensities. Symbols represent experimental data and solid lines represent theoretical fit.

Open aperture experimental data of BDDH for different excitation intensities are found to fit well for typical values of $\beta$ (see Table). Imaginary part of third-order susceptibilities $\left(\operatorname{Im} \chi^{(3)}\right)$, corresponding to $\beta$, are evaluated using Eq. (2) and given in Table. The nonlinear absorption of BDDH may include instantaneous two-photon type absorption and excited-state absorption (ESA)/re- 
verse saturable absorption (RSA). In general, in each of the wavelength region, the nonlinear absorption of the optical materials depends mainly on the laser-matter in- teraction time and cross-section area of excited levels. The nonlinear absorption of BDDH molecules can be explained by using a five-level model [31-33].

TABLE

Computed values of the excited-state absorption coefficient $\left(\sigma_{\text {exc }}\right)$, nonlinear absorption coefficient $(\beta)$, nonlinear refractive index $\left(n_{2}\right)$ and third-order susceptibility $\left|\chi^{(3)}\right|$ at different excitation intensities $\left(I_{0}\right)$.

\begin{tabular}{c|c|c|c|c|c|c}
\hline \hline $\begin{array}{c}\text { Excitation } \\
\text { intensity }\left(I_{0}\right) \\
{\left[\mathrm{GW} / \mathrm{cm}^{2}\right]}\end{array}$ & $\begin{array}{c}\sigma_{\text {exc }} \\
{\left[10^{-19} \mathrm{~cm}^{2}\right]}\end{array}$ & $\begin{array}{c}\beta \\
{\left[10^{-9} \mathrm{~cm} / \mathrm{W}\right]}\end{array}$ & $\begin{array}{c}\operatorname{Im} \chi^{(3)} \\
{\left[10^{-12} \mathrm{esu}\right]}\end{array}$ & $\begin{array}{c}n_{2} \\
{\left[10^{-14} \mathrm{~cm}^{2} / \mathrm{W}\right]}\end{array}$ & $\begin{array}{c}\operatorname{Re} \chi^{(3)} \\
{\left[10^{-12} \mathrm{esu}\right]}\end{array}$ & $\begin{array}{c}\left|\chi^{(3)}\right| \\
{\left[10^{-12} \mathrm{esu}\right]}\end{array}$ \\
\hline 0.4 & 16 & 5.65 & 1.31 & 4.72 & 2.59 & 2.90 \\
1.01 & 7.8 & 4.01 & 0.93 & 3.19 & 1.75 & 1.98 \\
2.14 & 4.35 & 3.36 & 0.78 & 2.60 & 1.42 & 1.62 \\
3.31 & 2.92 & 3.16 & 0.73 & 2.42 & 1.32 & 1.52
\end{tabular}

A typical energy level diagram of five-level model is shown in Fig. 5, in which $S_{0}, S_{1}$ and $S_{2}$ are: ground states, first singlet state and the next higher excited singlet state, respectively, whereas $T_{1}$ and $T_{2}$ are lower triplet state and next higher triplet state. Each of these states have a number of vibrational levels. When two photons of the same energy or different energies are absorbed simultaneously from the ground state $S_{0}$ to the first excited state $S_{1}$, then it is known as two-photon absorption (TPA), where in case of ESA, molecules are excited from an excited state $S_{1}$ to a higher excited state $S_{2}$. For this to happen, the population of the excited state ( $S_{1}$ and/or $T_{1}$ ) needs to be high enough, so that probability of photon absorption from that state is high. Here it is worth pointing out that a singlet transition does not deplete the population of first singlet state $S_{1}$ appreciably, since atoms excited to $S_{2}$ decay to $S_{1}$ itself within picoseconds. However, in nanosecond time scale, state $S_{1}$ electrons are transferred to state $T_{1}$ via intersystem crossing (ISC), from where transition to state $T_{2}$ (triplettriplet absorption) occurs [33].

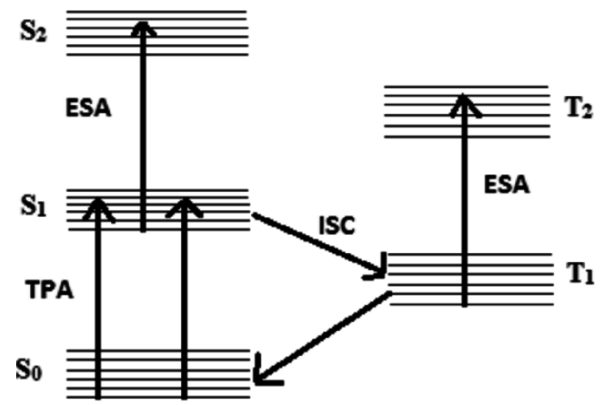

Fig. 5. Five-level energy diagram.

Furthermore, Fig. 4 depicts that minima in normalised transmittance occur around the focal plane, indicating RSA character, which implies ESA finally results in RSA in BDDH [33]. This feature can be confirmed by comparing magnitude of cross-section of the ground state $\left(\sigma_{\mathrm{g}}\right)$ and the cross-section of excited state $\left(\sigma_{\text {exc }}\right)$ of the sample. The values of $\sigma_{\text {exc }}$ for different excitation intensities were estimated by using Eq. (4b) and values are given in Table. Further, using Eq. (5) the value of ground state absorption cross-section $\left(\sigma_{\mathrm{g}}\right)$ is calculated and found to be $0.6 \times 10^{-19} \mathrm{~cm}^{2}$. A comparison between the ground state and the excited state absorption cross-section area manifests that $\sigma_{\text {exc }}>\sigma_{\mathrm{g}}$ which confirms RSA in BDDH.

The dependence of excitation intensity on nonlinear absorption coefficients is shown in Fig. 6. It is noticed that $\beta$ decreases gradually with increasing excitation intensity which is a consequence of sequential two-photon absorption via RSA $[34,35]$ and hence these results suggest that BDDH can be utilised as good reverse saturable absorber in visible region [15].

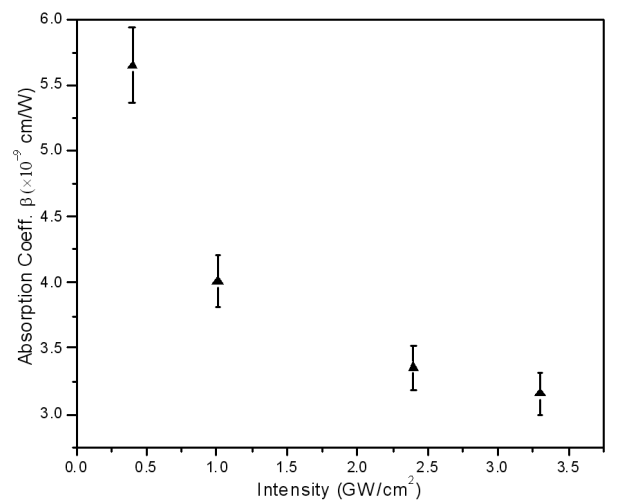

Fig. 6. Variation of excitation intensity $\left(I_{0}\right)$ vs. absorption coefficient $(\beta)$.

By closed aperture measurements, nonlinear refractive properties e.g. self-focusing/defocusing of the sample can be studied. The closed aperture scans of BDDH are shown in Fig. 7 for different excitation intensities 0.4, 
$1.01,2.14$ and $3.31 \mathrm{GW} / \mathrm{cm}^{2}$. In this figure, symbols represent experimental transmission data, while solid lines are obtained by fitting the experimental data to the nonlinear transmittance given by Eq. (6). Here, nonlinear refractive index $\left(n_{2}\right)$ is taken to be a fitting parameter.

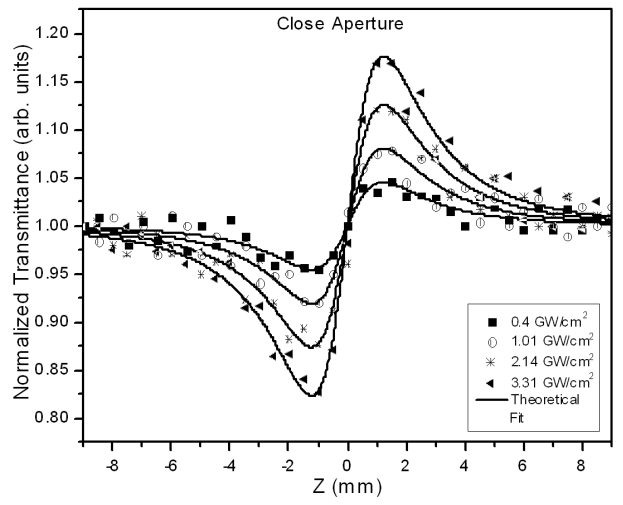

Fig. 7. Closed aperture measurements (at $532 \mathrm{~nm}$ ) of 2,3-butanedione dihydrazone at excitation intensities. Symbols represent experimental data and solid lines represent theoretical fit.

Close aperture experimental data of BDDH for different excitation intensities are found to fit well for typical values of $n_{2}$ (see Table). Real part of third-order susceptibilities $\left(\operatorname{Re} \chi^{(3)}\right)$, corresponding to $n_{2}$, is computed using Eq. (8) and given in Table. It is clearly evident from Fig. 7 that the sample exhibits prefocal valley and postfocal peak characteristics, which is direct indication of positive $n_{2}$ (positive lens) and it suggests BDDH can also be used as self-focusing materials around $532 \mathrm{~nm}$. In addition, the reliability of measurements of nonlinear refraction in BDDH is confirmed by conditions: (i) peak-valley character of BDDH satisfies the condition of $\Delta Z_{P-V} \sim 1.7 z_{0}$ (where $\Delta Z_{P-V}$ is the separation between the maxima and minima of the closed aperture curves) [22].

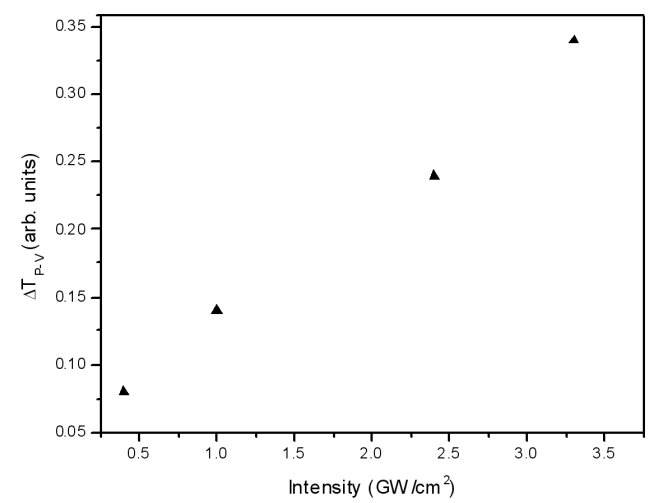

Fig. 8. Variation of excitation intensity $\left(I_{0}\right)$ vs. $\Delta T_{P-V}$.

Moreover, (ii) $\Delta T_{P-V}$ increases linearly with increasing excitation intensities (Fig. 8) of the laser. Inter- estingly, our reported values of nonlinear optical coefficients $\beta, n_{2}$ and $\chi^{(3)}$ (summarised in Table) are larger than the values measured in BDDH using third harmonic generation technique [36] and quoted for other conjugated organic materials $[37,38]$ under similar experimental conditions. Here it is worth mentioning that, in all cases, empty quartz cell, pure dimethyl sulphoxide (DMSO) and tetrahydrofuran (THF) were separately measured under the same experimental conditions and their magnitude of $\chi^{(3)}$ were found to be $\approx 10^{-15} \mathrm{esu}$, which is nearly $10^{3}$ smaller than the values reported in Table. Here reported values of nonlinear optical coefficients are of BDDH only.

\section{Conclusions}

2,3-butanedione dihydrazone compound was synthesised by chemical route. Its third-order nonlinear optical properties were investigated by $z$-scan technique with frequency doubled, $Q$-switched $5 \mathrm{~ns}$ Nd:YAG laser at wavelength $532 \mathrm{~nm}$ with repetition rate of $10 \mathrm{~Hz}$. The values of $\beta, n_{2}$ and $\chi^{(3)}$ of BDDH are obtained and noticed that these values are nearly ten times larger than the values reported elsewhere. The effect of excitation intensity on nonlinear optical properties of BDDH has been studied and found that with increasing excitation intensity nonlinear optical coefficients decrease. It is also noticed that BDDH exhibits reverse saturable absorption at $532 \mathrm{~nm}$ and this behaviour may be utilized for optical power limiting. Results also suggest that 2,3-butanedione dihydrazone can be one of the promising candidate materials for third-order nonlinearity based optical devices.

\section{References}

[1] C.S. Suchand Sandeep, A.K. Samal, T. Pardeep, Reji Philip, Chem. Phys. Lett. 485, 326 (2010).

[2] B.Y. Xu, Z. Liu, X. Zhang, Y. Wang, J. Tian, Y. Huang, Y. Ma, X. Zhang, Y. Chen, Adv. Mater. 21, 1275 (2009).

[3] J. Wang, W.J. Blau, J. Opt. A, Pure Appl. Opt. 11, 024001 (2009)

[4] Y.H. Lee, Y. Yan, L. Polavarapu, Qing-Huaxu, Appl. Phys. Lett. 95, 023105 (2009)

[5] T. Huang, Z. Hao, H. Gong, Z. Liu, S. Xiao, S. Li, Y. Zhai, S. You, I. Wang, J. Qn, Chem. Phys. Lett. 451, 213 (2008)

[6] K. Ogusu, J. Yamasake, S. Maeda, M. Kitao, M. Minakata, Opt. Lett. 29, 265 (2004)

[7] I. Polyzos, G. Tsigaridas, M. Fakis, V. Giannetas, P. Persephonis, J. Mikroyanidas, Chem. Phys. Lett. 369, 264 (2003)

[8] I. Polyzos, G. Tsigaridas, M. Fakis, V. Giannetas, P. Persephonis, Opt. Lett. 30, 2654 (2005).

[9] S. Maruo, O. Nakamura, S. Kawata, Opt. Lett. 22, $132(1997)$

[10] M. Singh, P. Aghamkar, N. Kishore, P.K. Sen, Opt. Laser Technol. 40, 215 (2008) 
[11] M. Singh, P. Aghamkar, Opt. Commun. 281, 1251 (2008)

[12] P. Aghamkar, M. Singh, J. Mod. Opt. 55, 931 (2008).

[13] H.S. Nalwa, S. Miyata, Nonlinear Optics of Organic Molecules and Polymers, CRC, Boca Raton (FL) 1997.

[14] H.S. Nalwa, in: Handbook of Organic Conductive Molecules and Polymers, Vol. 4, Wiley, Hoboken 1997, p. 261.

[15] P. Poornesh, G. Umesh, P.K. Hegde, M.G. Manjunatha, K.B. Man, A.V. Adhikari, Appl. Phys. B 97, $117(2009)$

[16] S. Yin, H. Xu, W. Shi, Y. Gao, Y. Song, J. Wing, Y. Lom, B.Z. Taj, Polymer 46, 7670 (2005)

[17] U. Gublor, S. Concilio, C. Bosshard, I. Biaggio, P. Gunther, R.E. Martin, M.S. Edelmann, J.A. Wykto, F. Diederich, Appl. Phys. Lett. 81, 2322 (2002)

[18] W.S. Fann, S. Benson, J.M.J. Madey, S. Etemad, G.L. Baker, F. Kajzar, Phys. Rev. Lett. 62, 1492 (1989)

[19] F. Krausz, E. Winter, G. Leising, Phys. Rev. B 39, 3701 (1989)

[20] H.S. Nalwa, A. Kakuta, A. Mukoh, J. Appl. Phys. 73, 4743 (1993)

[21] R.L. Sutherlands, Handbook of Nonlinear Optics, Marcel Dekker, New York 2003, Ch. 8.

[22] M. Sheik Bahae, E.W. Van Stryland, IEEE J. Quant. Electron. 26, 760 (1990)

[23] H. Wang, Z. Li, P. Shao, J. Qin, Z. Huang, J. Phys. Chem. B 114, 22 (2010)

[24] P. Poornesh, G. Umesh, P.K. Hegde, M.G. Manjunatha, K.B. Man, A.V. Adhikari, Opt. Laser Technol. 42, 230 (2010)

[25] N. Li, J. Lu, X. Xia, Q. Xu, L. Wang, Polymer 50, $428(2009)$
[26] C.R. Hauer, G.S. King, E.L. McCool, W.B. Euler, J.D. Ferrara, W.J. Youngs, J. Am. Chem. Soc. 109, 5760 (1987)

[27] E.W. van Stryland, M. Sheik Bahae, in: Z-Scan Measurement of Optical Nonlinearities, Characterization Techniques and Tabulation for Organic Nonlinear Materials, Eds. M.G. Kuyzk, C.W. Dirk, Marcel Dekker, Inc., 1998, p. 655.

[28] F. Li, Y. Song, K. Yang, S. Liu, C. Li, Appl. Phys. Lett. 71, 2073 (1997)

[29] R. Rangel-Rojo, S. Yamada, H. Matsuda, D. Yankelevich, Appl. Phys. Lett. 72, 1021 (1998).

[30] B.C. Sherman, B.K. Schmitz, W.B. Wuler, Chem. Mater. 7, 806 (1995)

[31] W.E. Douglas, L.G. Klapshina, A.N. Rubinov, G.A. Domrashev, B.A. Bushuk, J.A. Kalvinkovskaya, Proc. SPIE 4106, 360 (2000)

[32] W. Feng, W. Yi, H. Wu, M. Ozaki, K. Yashino, J. Appl. Phys. 98, 034301 (2005)

[33] F.Z. Henari, J. Opt. A, Pure Appl. Opt. 3, 188 (2001)

[34] S. Couris, E. Koudoumas, A.A. Ruth, S. Leach, J. Phys. B, At. Mol. Opt. Phys. 28, 4537 (1995)

[35] C.V. Bindu, S.S. Harilal, V.P.N. Nampoori, C.P.G. Vallabhan, Appl. Phys. B 70, 429 (2000)

[36] H.S. Nalwa, T. Hamada, A. Kakuta, A. Mukoh, Synth. Met. 57, 3901 (1993)

[37] Y. Cheng, T. He, H. Hao, S. Zhu, H. Xiao, Opt. Commun. 282, 4271 (2009).

[38] U. Tripathy, P.B. Bisht, Opt. Commun. 261, 353 (2006) 